obtained for r-FSH was €82.3 (€78.3-€98.1) and €73.3 (€67.6-€81.7) for $\mathrm{u}-\mathrm{FSH}$, so the ICER in mature oocytes obtained was 4.7 (4.0-15.2).

Conclusions According to scientific evidence r-FSH appears to be more effective in women undergoing $\mathrm{COH}$; however, this slight increase in efficacy does not seem to compensate for the difference in price, the result being that u-FSH is more cost effective.

No conflict of interest.

\section{OHP-067 PRESCRIPTION PROFILE ANALYSIS OF PROTON PUMP INHIBITORS IN A TERTIARY HOSPITAL}

doi:10.1136/ejhpharm-2013-000276.440

A Díez Alcántara, V Saavedra Quirós, E Santiago Prieto, A Torralba Arranz. Hospital Universitario Puerta de Hierro, Pharmacy, Madrid, Spain

Background In October 2011, selection criteria for proton pump inhibitors (PPIs) were published, recommending the use of omeprazole as a drug of choice because, at equipotent doses, it is the most cost-effective drug, compared to other PPIs.

Purpose To describe the prescription profile of PPIs for different consultants and in patients who are discharged from hospital.

Materials and Methods Information about the prescriptions for PPIs issued during 2011 was obtained from the pharmaceutical software. The data were analysed and classified according to therapeutic group, active principle, number of defined daily doses (DDDs), service and number of prescriptions. The percentage DDD of each active principle with respect to the PPI group as a whole was also investigated.

Results During 2011, 9.654 prescriptions were written. Gastroenterology was the Medical Service with the most prescriptions, followed by Internal Medicine and Otolaryngology. The percentage DDD of each PPI prescribed in each service, related to the whole of the PPIs was:

- Gastroenterology: 26\% omeprazole; $14 \%$ pantoprazole; 18\% lansoprazole; $16 \%$ esomeprazole; $26 \%$ rabeprazole. Total, 2213 prescriptions.

- Otolaryngology: $55 \%$ omeprazole; $22 \%$ pantoprazole; $2 \%$ lansoprazole; $7 \%$ esomeprazole; $14 \%$ rabeprazole. Total, 1074 prescriptions.

- Internal Medicine: $82 \%$ omeprazole; $7 \%$ pantoprazole; $0,5 \%$ lansoprazole; $10 \%$ esomeprazole; $0,5 \%$ rabeprazole. Total, 619 prescriptions.

Conclusions Omeprazole is the PPI with the highest percentage of DDD prescribed, nevertheless prescriptions for it are on the low side (less than 30\% in Gastroenterology and Otolaryngology, and less than $85 \%$ in Internal Medicine); this means that there is still a lot more room for improvement. The Service which made the best selection of IPPs was Internal Medicine, followed by Otolaryngology, and finally Gastroenterology.

Despite the low number of prescriptions made in hospital, compared to the ones prescribed in Primary Care, there is still a lot of work to be done to improve the selection of IPPs prescribed in hospital.

No conflict of interest.

\section{OHP-068 REPEAT AUDIT OF LMWH USE IN THROMBOPROPHYLAXIS ON AN ORTHOPAEDIC SURGERY WARD, EMERGENCY CENTRE, BELGRADE}

doi:10.1136/ejhpharm-2013-000276.441

M Kara-Jovanovi, RD Rajinac, JG Jevtic, SLJ Stojicevic, KM Klancnik. Clinical Center Serbia, Hospital pharmacy Emergency Center, Belgrade, Serbia

Background The Guideline for Prevention of Deep Venous Thromboembolism in Orthopedic Surgery, based on current European and American Guidelines, was introduced in late 2009 on the Orthopedic Surgery Ward, Emergency Centre, Belgrade.
Hospital pharmacists were actively involved in writing, and monitoring the implementation of, the guideline.

Purpose The first audit of implementation of the guideline was in March 2010. The aim was to show if all patients were receiving thromboprophylaxis according to the guideline; and whether thromboprophylaxis was being recommended for patients after discharge from hospital.

The aim of the repeat study was to estimate if there were differences in implementing the guideline.

Materials and Methods Monitoring of prescriptions for patients in hospital and recommendations for thromboprophylaxis on the discharge documentation.

This study covered the period from January to March 2012 and compared results with the same period in 2010.

Results 2010 year: Total number of patients 104; 97\% of patients received the recommended anticoagulant treatment during hospitalisation, and $85 \%$ patients received the recommended anticoagulant treatment after hospitalisation.

2012 year: Total number of patients 143; 96.5\% of patients received the recommended anticoagulant treatment during hospitalisation, and $91.5 \%$ of patients received the recommended anticoagulant treatment after hospitalisation.

Conclusions During the monitoring period 3\% of patients did not receive the recommended thromboprophylaxis during hospitalisation in 2010 , and $3.5 \%$ of patients in 2012 .

By continuous monitoring of recommended thromboprophylaxis after release from hospital, it was concluded that $15 \%$ of patients failed to receive the recommended treatment in 2010, and $8.4 \%$ in 2012 .

The repeat audit two years later showed a similar percentage of thromboprophylaxis prescribing during hospitalisation, and significant improvement in thromboprophylaxis recommendations at the discharge of patients from hospital.

No conflict of interest.

\section{OHP-069 RETROSPECTIVE STUDY ABOUT PATIENTS WITH A STOMA AND THEIR NEEDS IN THE HEALTH DISTRICT OF PATTI (MESSINA)}

doi:10.1136/ejhpharm-2013-000276.442

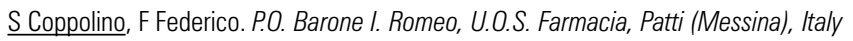

Background Serious conditions of the bowel and bladder often require the formation of a stoma. It is estimated that 650,000 people in Europe live with a stoma. A person with a stoma not only needs post-operative medical care, but also appliances and accessories to increase his quality of life. In the Local Health Authority (LHA) of Messina appliances and accessories are given, for free, every month by the Hospital/District Pharmacies.

Purpose To point out, for the Health District (HD) of Patti, which is one of seven HDs in the LHA of Messina, the number of patients with a stoma, the types of appliances and accessories used, and with what difficulties we assist patients.

Materials and Methods Through the retrospective interrogation of an administrative database it was possible to assess the number of patients with a stoma who live in the HD of Patti and, particularly, those who received appliances and accessories from 01/01/2011 to $31 / 12 / 2011$. For each patient the gender, age, kind of stoma, type and amount of appliances and accessories were recorded. All data gathered were analysed with 'Statistica' software. During the delivery of devices patients were also interviewed about problems they had experienced. All answers were collected and reviewed anonymously.

Results 70 patients were recorded, mainly with a colostomy. The incidence in the HD population was $1: 715$ inhabitants. $55.7 \%$ of patients were males and the average age was 74.3 years $[39,94]$. 
There was no difference between the number of patients with a one or a two-piece appliance. Closed appliances are most commonly used; $75 \%$ of patients had experienced at least one appliance leakage/year; $57.15 \%$ needed adhesive paste, $36 \%$ a skin protection barrier. Only $10 \%$ had difficulty in removing the stoma appliance causing skin stripping and ulceration.

Conclusions In the Patti HD hospital pharmacists can play a role in supporting and guiding patients and their carers by providing information, education and counselling on how to best manage their stoma in daily activities, in order to achieve an acceptable quality of life.

No conflict of interest.

\section{OHP-070 SATISFACTION WITH ELECTRONIC PRESCRIBING IN A GENERAL HOSPITAL}

doi:10.1136/ejhpharm-2013-000276.443

'T Sánchez Casanueva, ${ }^{2} \mathrm{P}$ López Sánchez, ${ }^{2} E$ Jerez Fernández, ' $\mathrm{JJ}$ Márquez Nieves, ${ }^{2} \mathrm{M}$ Heredia Benito. 'Complejo Hospitalario La Mancha Centro, Pharmacy, Tomelloso, Spain; ${ }^{2}$ Complejo Hospitalario La Mancha Centro, Pharmacy, Alcázar de San Juan, Spain

Background Electronic prescribing (EP) is a useful tool for improving the safety and adaptability of the prescription process. Surveys enable us to find out the satisfaction of users and potential areas for improvement.

Purpose To find out how satisfied doctors and nurses of the Internal Medicine Service (IMS) were with EP.

Materials and Methods In 2010 the 'Mambrino XXI' electronic medical record, which is an EP module, was implemented in a 100bed general hospital.

In 2012 the Pharmacy Service developed an anonymous and confidential survey that was given to the doctors and nurses of the ISM. The questionnaire included 6 questions rated with a Likert scale (1: very bad/strongly disagree, 2 : bad/disagree, 3: Regular/indifferent; 4: good/agree, 5: very good/strongly agree): 1 . How do you consider the ease of use? 2. How does the speed of the application seem to you? 3. Are the alerts for allergies and duplications useful? 4. Do you think it prevents medication errors and improves safety? 5. Is the design of printed orders satisfactory? 6. What do you think about the support from the Pharmacy Service?

An overall satisfaction question was also included with 4 answers: very satisfied, satisfied, dissatisfied, very dissatisfied.

Results 6 doctors and 10 nurses completed the questionnaire. The average score was 3.7 for question $1 ; 2.9$ for question $2 ; 3.9$ for question 3; 3 for question 4; 3.1 for question 5 and 4.1 for question 6; 6 respondents were very satisfied, 5 satisfied and 5 dissatisfied.

Conclusions The survey evaluated aspects of practise use, safety and Pharmacy Service support. More than two-thirds of doctors and nurses of the ISM were satisfied with the EP. There are opportunities for improve all the aspects investigated, especially the programme speed, the perceived safety and the design of printed medical orders.

No conflict of interest.

\section{OHP-071 STUDY OF GLUTAMINE USE IN ADULT PARENTERAL NUTRITION}

doi:10.1136/ejhpharm-2013-000276.444

R Tamayo Bermejo, C Gallego Fernández, J González Chávez, M Ruiz de Villegas, I Muñoz Castillo. HRU CARLOS HAYA, Servicio de Farmacia, Malaga, Spain

Background Intravenous glutamine supplementation in patients with catabolic stress is widespread in clinical practise, although there is no clear consensus on its use.
Purpose To study the use of glutamine in adult parenteral nutrition to adapt it to the available scientific evidence and to assess the economic impact of parenteral nutritional treatment.

Materials and Methods Retrospective observational study of units of parenteral nutrition (PNU) produced during 2011.

We studied the three services that used PN most: Digestive Surgery, Digestive and Intensive Care Unit (ICU).

Data collection source: Software in parenteral nutrition area. Pharmacy Management System.

Study Variables:

Protocols produced by service (number of each PNU protocol, protocol type, number of patients with each protocol and duration of nutrition)

Individualized PNUs produced by service (number of PNUs, number of patients and duration of $\mathrm{PN}$ )

Cost of each protocol and glutamine cost therein.

Results Of all adult PNUs produced in accordance with a protocol, $58 \%$ were stress protocols.

PNU per service (including individual):

Digestive Surgery: $80 \%$ of the total number of PNUs were stress PNU and corresponded to $68 \%$ of the patients. There is scientific evidence to recommend the use of glutamine in patients undergoing major abdominal surgery.

Digestive: $52 \%$ of the total number of PNUs were stress PNU and corresponded to $54 \%$ of the patients. Glutamine use was associated with acute pancreatitis and inflammatory disease, although clinical studies are insufficient to recommend this.

ICU: $63 \%$ of the total number of PNUs were stress PNU and corresponded to $72 \%$ of patients. There is evidence of clinical benefit with high recommendation.

Glutamine cost varies between $45.4 \%-55.7 \%$ of the total cost per PNU.

$76.5 \%$ of the total cost of protocolized PNUs corresponded to stress protocols.

Conclusions An opportunity for improvement is identified in the use of glutamine. We propose a detailed study of the prescription/ indication to rationalise its high use and associated costs.

No conflict of interest.

\section{OHP-072 STUDY OF USE OF COLISTIN IN A SERBIAN CLINICAL CENTRE}

doi:10.1136/ejhpharm-2013-000276.445

M Grcic, M Tomic. Clinical Center Serbia, Service for the Pharmaceutical Business and Supply, Belgrade, Serbia

Background Colistin (polymyxin E) is a mixture of cyclic polypeptides colistin A and B and it remains one of the last-resort antibiotics for multidrug resistant species of Pseudomonas and Acinetobacter. The increased use of Colistin was noticed at the end of 2011.

Purpose To analyse the use of Colistin due to increased bacterial resistance and difficulties in supply, as no licence has been issued for marketing authorization of this medicine in Serbia.

Materials and Methods A retrospective descriptive study of patients who started Colistin treatment from January to September 2012. We reviewed those forms that recorded: patient demographic data, posology, duration, kind of treatment and type of infection. All data were collected in an Excel database.

Results In this period, 86 patients were prescribed Colistin $(55 \%$ men). In $74 \%$ cases the posology was $1 \mathrm{M} \mathrm{IU} / 8 \mathrm{~h}$, and in $26 \%$ was $2 \mathrm{M} \mathrm{IU} / 8 \mathrm{~h}$; mean duration of treatment was 18.07 days, but in $38 \%$ patients we did not get data about duration of treatment. Colistin 